\title{
Dysregulated microRNA expression profiles in gastric cancer cells with high peritoneal metastatic potential
}

\author{
YANING FENG $^{1 *}$, FEIHU BAI $^{1 *}$, YANJIE YOU $^{1 *}$, FANGYUN BAI $^{2}$, \\ CHUANXIA WU ${ }^{3}$, RUIJUAN XIN ${ }^{1}$, XUE LI $^{1}$ and YONGZHAN NIE ${ }^{4}$
}

\begin{abstract}
${ }^{1}$ Department of Gastroenterology, Ningxia Hui Autonomous Region People's Hospital, Yinchuan, Ningxia Hui Autonomous Region 750021; ${ }^{2}$ Department of General Medicine, General Hospital of Ningxia Medical University, Yinchuan, Ningxia Hui Autonomous Region 750001; ${ }^{3}$ Department of Gastroenterology, The Second People's Hospital of Linyi, Linyi, Shandong 276000; ${ }^{4}$ Department of Gastroenterology, Xijing Hospital of Digestive Diseases, State Key Laboratory of Cancer Biology and Institute of Digestive Diseases, Xi'an, Shaanxi 710000, P.R. China
\end{abstract}

Received April 8, 2018; Accepted August 14, 2018

DOI: 10.3892/etm.2018.6783

\begin{abstract}
Despite significant developments in its clinical treatment, the reported incidence and mortality of gastric cancer have exhibited marked increases. The molecular mechanisms of gastric cancer initiation and progression remain to be fully elucidated. The aim of the present study was to identify novel microRNAs (miRNAs/miRs) with a role in the peritoneal metastasis of gastric cancer by comparing the miRNA expression in the gastric cancer cell line GC9811 with that in its variant GC9811-P, a sub-cell line with a high potential for peritoneal metastasis. A miRNA microarray analysis identified 153 dysregulated miRNAs, including 74 upregulated and 79 downregulated miRNAs. Of these, four significantly upregulated miRNAs (miR-181a-5p, miR-106b-5p, miR-199a-3p and miR-148a-3p) and four downregulated miRNAs (miR-146a-5p, miR-21-5p, miR-222-3p and miR-221-3p) were selected and further confirmed by reverse transcription-quantitative polymerase chain reaction analysis. Furthermore, knockdown of miR-21-5p promoted the migration and invasion of GC9811 cells. Collectively, the results suggested that the miRNA expression profile in GC9811-P vs. GC9811 cells was altered to favor disease progression, and the dysregulated miRNAs, including miR-21-5p, may therefore provide novel biomarkers and potential therapeutic targets for gastric cancer metastasis.
\end{abstract}

Correspondence to: Dr Yongzhan Nie, Department of Gastroenterology, Xijing Hospital of Digestive Diseases, State Key Laboratory of Cancer Biology and Institute of Digestive Diseases, 169 Changlexi West Road, Beilin, Xi'an, Shaanxi 710000, P.R. China E-mail: yzn448983360@126.com

${ }^{*}$ Contributed equally

Key words: microarray, microRNA expression, gastric cancer, peritoneal metastasis

\section{Introduction}

Gastric cancer is the fourth most common malignancy and the second leading cause of cancer-associated mortality worldwide (1). An estimated $60 \%$ of patients with advanced gastric cancer succumb to peritoneal metastasis, which accounts for $50 \%$ of recurrences, thus making it the leading cause of mortality associated with this disease $(2,3)$. Peritoneal metastasis may be regarded as a complex and multi-step process that consists of a series of independent stages, whereby cancer cells migrate from the primary neoplasm to a distant location. To date, the mechanisms of peritoneal metastasis of gastric cancer have remained elusive, and molecular markers for gastric cancer metastasis and tumor progression remain to be identified. Data from available studies are difficult to interpret due to the complexity arising from tumor heterogeneity. Thus, identification of molecular and biological alterations that occur during the peritoneal metastasis of gastric cancer may facilitate the investigation of the pathology of the disease and lead to the discovery of novel prognostic markers to more accurately predict clinical outcomes and to individualize treatments for patients with gastric cancer.

MicroRNAs (miRNAs/miRs) are a series of non-coding RNAs of 19-25 nt in length that regulate gene expression at the post-transcriptional level $(4,5)$. miRNAs may silence their target genes by inhibiting mRNA translation or degrading the mRNA molecules by binding to their 3 '-untranslated region, thereby having a crucial role in cancer biology. Abundant evidence has demonstrated that dysregulated expression of certain miRNAs is involved in cancer progression and metastasis, and that these miRNAs may serve as novel biomarkers or therapeutic targets (6-8). With the recent development of miRNA detection techniques, identification of miRNAs associated with cancer progression/metastasis may complement and enhance the current understanding of the origins and evolution of peritoneal metastasis, and may revolutionize the pre-operative prediction of the status of peritoneal metastases from gastric cancer. However, despite such great promise, the role of miRNAs in gastric cancer, particularly during peritoneal metastasis, remains to be elucidated. Therefore, in 
the present study, miRNA expression profiles were examined in gastric cancer cell lines with different metastatic potential by miRNA microarray analysis. The biological functions of miR-21-5p, a miRNA whose downregulation was identified to be associated with peritoneal metastasis, were subsequently examined by cell migration and invasion assays.

\section{Materials and methods}

Cell lines and culture. The human gastric cancer cell line GC9811 and its derived sub-cell line GC9811-P with high peritoneal metastatic potential were obtained from the State Key Laboratory of Cancer Biology at Xijing Hospital of Digestive Diseases (The Fourth Military Medical University, Xi'an, China). The GC9811 cell line was originally purchased from American Type Culture Collection (Manassas, VA, USA). The GC9811-P cell line was established and characterized as having a high metastatic potential in the peritoneum by orthotopic tumor cell implantation (9). Cells were cultured in RPMI-1640 medium supplemented with $10 \%$ fetal bovine serum (FBS; both Gibco; Thermo Fisher Scientific, Inc. Thermo Fisher Scientific, Inc., Waltham, MA, USA), 100 units $/ \mathrm{ml}$ penicillin and $0.1 \mathrm{mg} / \mathrm{ml}$ streptomycin (both Sigma-Aldrich; Merck KGaA, Darmstadt, Germany) in 6-well plates (Corning Costar; Corning Inc., Corning, $\mathrm{NY}, \mathrm{USA}$ ) at $37^{\circ} \mathrm{C}$ in a humidified atmosphere containing $5 \% \mathrm{CO}_{2}$.

RNA extraction and small RNA isolation. When the cells reached $80-90 \%$ confluence, they were washed with ice-cold PBS three times and homogenized immediately in TRK-100 lysis solution (LC Sciences, Houston, TX, USA). Small RNA was extracted from each sample using an RNAiso for Small RNA kit (cat. no. 9753A; Takara Biotechnology Co., Ltd., Dalian, China) according to the manufacturer's protocol with certain modifications; the extracted small RNA was resuspended and incubated at $-80^{\circ} \mathrm{C}$ overnight to enhance the precipitation efficiency of RNAs with a low molecular weight (20-200 nt). The concentration and purity of the small RNA were determined with an ultraviolet spectrophotometer at 260 and $280 \mathrm{~nm}$. Only the RNA samples with a 260/280 nm absorbance ratio of $>1.8$ were used for the subsequent analysis.

miRNA microarray assay. Microarray assays were performed by an external service provider (LC Sciences). The assay began with a 4-8- $\mu$ g total RNA sample, which was 3'-extended with a poly(A) tail using poly(A) polymerase. An oligonucleotide tag was ligated to the poly(A) tail for subsequent fluorescent dye staining. Hybridization was performed overnight on a $\mu$ Paraflo microfluidic chip using a micro-circulation pump (both Atactic Technologies, Inc., Houston, TX, USA). On the microfluidic chip, each detection probe consisted of a chemically modified nucleotide-coding segment complementary to the target miRNA (from miRbase; http://www.mirbase.org/) or other RNA (control or customer-defined sequences), and a spacer segment of polyethylene glycol to extend the coding segment away from the substrate. The detection probes were generated by in situ synthesis using a PCR-generated template method. The melting temperatures of hybridized products were balanced by chemical modifications of the detection probes. Hybridization
Table I. Differentially expressed miRNAs detected by microarray analysis.

\begin{tabular}{lcc}
\hline miRNA & Fold change & P-value \\
\hline miR-146a-5p & -208.3 & $2.70 \times 10^{-3}$ \\
miR-21-5p & -10.48 & $7.40 \times 10^{-3}$ \\
miR-221-3p & -13.98 & $1.26 \times 10^{-2}$ \\
miR-222-3p & -9.74 & $1.01 \times 10^{-2}$ \\
miR-181a-5p & 13.20 & $4.28 \times 10^{-3}$ \\
miR-106b-5p & 7.02 & $5.98 \times 10^{-3}$ \\
miR-199a-3p & 14.52 & $6.19 \times 10^{-3}$ \\
miR-148a-3p & 85.44 & $6.49 \times 10^{-3}$ \\
\hline
\end{tabular}

miRNA/miR, microRNA.

was performed using 1001 formamide solution $(25 \%)$ in $6 \mathrm{X}$ buffer (0.90 M NaCl, 60 mM Na${ }_{2} \mathrm{HPO}_{4}, 6$ mM EDTA; pH 6.8) at $34^{\circ} \mathrm{C}$ for $1 \mathrm{~h}$. Following RNA hybridization, tag-conjugating $\mathrm{Cy} 3$ dye was circulated through the microfluidic chip for dye staining. Fluorescence images were generated using a laser scanner (GenePix 4000B; Molecular Devices LLC, Sunnyvale, CA, USA) and digitized using Array-Pro 3.0 image analysis software (Media Cybernetics, Inc., Rockville, MD, USA). Data were analyzed by first subtracting the background and subsequently normalizing the signals using locally-weighted scatter plot smoothing regression.

Reverse transcription-quantitative polymerase chain reaction $(R T-q P C R)$. miRNA quantification was performed by RT-qPCR analysis. A total of $40 \mathrm{ng}$ total RNA containing miRNA was polyadenylated by poly(A) polymerase (Takara Biotechnology Co., Ltd.) and reverse transcribed to complementary (c)DNA using a PrimeScript miRNA cDNA Synthesis kit (Takara Biotechnology Co., Ltd.), according to the manufacturer's protocol. qPCR was performed using the miScript SYBR-Green PCR kit (Takara Biotechnology, Co., Ltd.) with the included miScript Universal primer and the miRNA-specific forward primers in an ABI PRISM 7900 Real-time PCR system (Applied Biosystems; Thermo Fisher Scientific, Inc.). The miRNA-specific primer sequences were miR-146a-5p, 5'-CGGTGAGAACTGAATTCCATGGGTT-3'; miR-181a-5p, 5'-AACATTCAACGCTGTCGGTGAGT-3'; miR-106b-5p, 5'-CGGTAAAGTGCTGACAGTGCACGAT-3'; miR-199a-3p, 5'-CGGACAGTAGTCTGCACATTGGTTA-3'; miR-148a-3p, 5'-CGGTCAGTGCACTACAAGAACTTT GT-3'; miR-21-5p, 5'-CGGTAGCTTATCAGACTGATGTTG A-3'; miR-222-3p, 5'-AGCTACATCTGGCTACTGGGTC-3'; and miR-221-3p, 5'-AGCTACATTGTCTGCTGGGTTTC-3'. Sequences were designed based on the miRNA sequences obtained from the miRBase database (http://microrna.sanger. ac.uk/). Each reaction was performed in a final volume of $20 \mu \mathrm{l}$ containing $2 \mu \mathrm{l} \mathrm{cDNA}, 0.5 \mathrm{mmol} / \mathrm{l}$ of each primer and SYBR-Green PCR Master mix (Takara Biotechnology Co., Ltd.). Following an initial incubation at $95^{\circ} \mathrm{C}$ for $15 \mathrm{~min}$, 40 cycles of denaturation at $94^{\circ} \mathrm{C}$ for $15 \mathrm{sec}$, annealing at $55^{\circ} \mathrm{C}$ for $30 \mathrm{sec}$ and extension at $70^{\circ} \mathrm{C}$ for $30 \mathrm{sec}$ were performed. PCRs were performed in triplicate for each sample and 
experiments were repeated a minimum of three times. At the end of the PCR cycles, melting curve analyses were performed in addition to electrophoresis of the products on 3.5\% agarose gels. U6 small nuclear RNA 6, pseudogene was used as an endogenous control (primers: U6 stem-loop forward, 5'-AGC GGGAAATCGTGCGTGACA-3' and reverse, 5'-GTGGAC TFGGGAGAGGACTGG-3). Relative expression of the target gene was calculated using the formula $R Q=2^{-\Delta \Delta C t}$ as described previously (10).

Lentiviral transfection. To silence miR-21-5p expression, validated specific small interfering RNA oligonucleotides (5'-UCAACAUCAGUCUGAUAAGCUA-3') were cloned into the lentiviral GV369/GV280 vector (GeneChem Inc., Shanghai, China). Lentiviral vector construction and transfection were performed according to the manufacturer's protocols. Cells were seeded in antibiotic-free medium at 1 day prior to transfection using Lipofectamine 2000 reagent (GE Healthcare Life Sciences, Little Chalfont, UK). At 24 and $48 \mathrm{~h}$ after transfection, cells were collected and the transfection and gene silencing efficiency was determined by detecting the fluorescence intensity of green fluorescent protein and RT-qPCR analysis, respectively.

Cell invasion assay. In vitro invasion assays were performed using 24-well Transwell chambers (Corning, Inc.) as described previously $(11,12)$. Matrigel (BD Biosciences, Franklin Lakes, NJ, USA) was diluted 1:8 with cold serum-free DMEM and coated onto the Transwell inserts. Cells were plated into the upper chambers at $2 \times 10^{4}$ cells per well in $100 \mu 1$ serum-free DMEM. The bottom chamber was filled with DMEM containing $10 \%$ FBS. Following incubation at $37^{\circ} \mathrm{C}$ for $24 \mathrm{~h}$, non-invasive cells in the upper chamber were removed. Invasive cells on the lower surface of the inserts were fixed with $4 \%$ paraformaldehyde at room temperature for $30 \mathrm{~min}$, stained with $0.2 \%$ crystal violet at room temperature for $2 \mathrm{~h}$ and counted under a light microscope (magnification, x100). Each experiment was performed three times in triplicate wells.

Wound healing assay. In brief, cells were seeded in 6-well plates at a density of $2 \times 10^{5}$ per well. After $12 \mathrm{~h}$, cells achieved $100 \%$ confluence. Two parallel line-shaped wounds of $1 \mathrm{~mm}$ in width were generated using $200 \mu \mathrm{l}$ pipette microtips (Corning, Inc.). Subsequently, the cells were washed with PBS twice and cultured in serum-free medium at $37^{\circ} \mathrm{C}$. The size of the wound was measured under phase-contrast microscopy (Carl Zeiss AG, Oberkochen, Germany) at 0 and $24 \mathrm{~h}$ post-wounding.

Statistical analysis. Statistical analysis was performed using GraphPad Prism version 5.02 (GraphPad Software, Inc., La Jolla, CA, USA). Student's t-tests, and one-way analysis of variance followed by the Student-Newman-Keuls post-hoc test were employed. $\mathrm{P}<0.05$ was considered to indicate a statistically significant difference.

\section{Results}

Deregulated miRNAs associated with the peritoneal metastatic potential of gastric cancer cells. The initial step of the present study was to search for miRNAs that are potentially involved in the peritoneal metastasis of gastric cancer cells by means of a microarray analysis. By using the expression levels of miRNAs in GC9811 cells as a control, 153 miRNAs were identified to be differentially expressed in GC9811-P cells with high metastatic potential via hierarchical clustering analysis, including 74 upregulated miRNAs and 79 downregulated miRNAs (Fig. 1A). The absolute value of the fold change was defined as $\geq 2$. Among the differentially expressed miRNAs, 67 had a signal intensity of $>500$. Of the 13 miRNAs obtained based on the criteria of a signal intensity of $>500$ and $\mathrm{P} \leq 0.01$ (Fig. 1B), 8 miRNAs with significant fold changes were selected for further analysis, comprising 4 downregulated miRNAs (miR-146a-5p, miR-21-5p, miR-221-3p and miR-222-3p) and 4 upregulated miRNAs (miR-181a-5p, miR106b-5p, miR-199a-3p and miR-148a-3p; Table I). The expression levels of these miRNAs were confirmed in GC9811 and GC9811-P cells by RT-qPCR analysis (Fig. 2).

Knockdown of miR-21-5p promotes the migration of GC9811 cells. Based on the above data, miR-21-5p was selected for further analysis. Lentiviral transfection was performed to achieve knockdown of miR-21-5p in GC9811 cells, in which miR-21-5p expression was increased compared with that in GC9811-P cells. Following $72 \mathrm{~h}$ of transfection followed by puromycin selection, RT-qPCR analysis confirmed that the expression levels of miR-21-5p were significantly downregulated in GC9811 cells (Fig. 3). To clarify the functions of miR-21-5p in gastric cancer progression and metastasis, the effect of miR-21-5p knockdown on GC9811 cell migration and invasion was examined. Confluent GC9811 cells with or without knockdown of miR-21-5p were scratched and cell migration was observed. It was observed that GC9811 cells with miR-21-5p knockdown exhibited a statistically significantly accelerated closure of the wound area compared with that of the empty vector-infected control cells (Fig. 4).

Knockdown of miR-21-5p elevates the invasiveness of GC9811 cells. Matrigel invasion assays were subsequently performed to confirm the effect of miR-21-5p on the metastatic progression of gastric cancer cells. As expected, it was observed that GC9811 cells with miR-21-5p knockdown exhibited a significant increase in their invasive capacity compared with that of the vector-transfected control cells (Fig. 5).

\section{Discussion}

Accumulating evidence suggests that miRNAs serve important roles as either oncogenes or tumor suppressor genes in the initiation and progression of cancer. The prognostic and therapeutic potential of a number of miRNAs that have been examined in various cancer types, including gastric cancer (13), but remain insufficiently elucidated. In particular, studies examining the miRNA expression status specifically associated with the peritoneal metastasis of gastric cancer are currently lacking. The principal reasons for this include unavailability or difficulty in obtaining model systems, including proper cell lines and gene-engineered animals. The present study focused on the miRNA expression profiles in a pair of gastric cancer cell lines with different peritoneal 
A
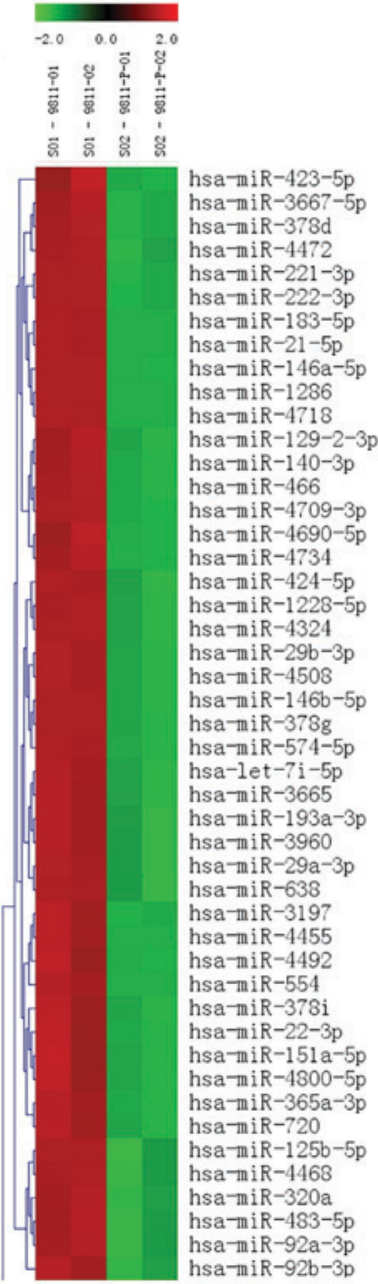

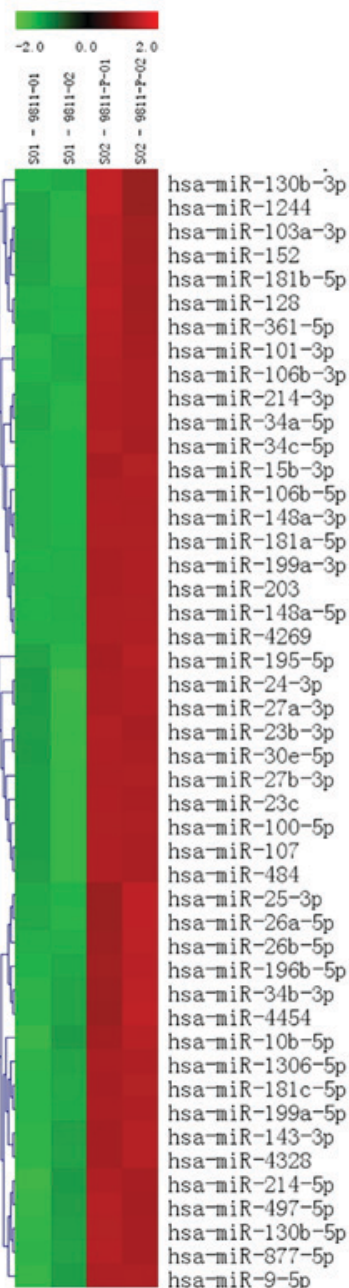

B

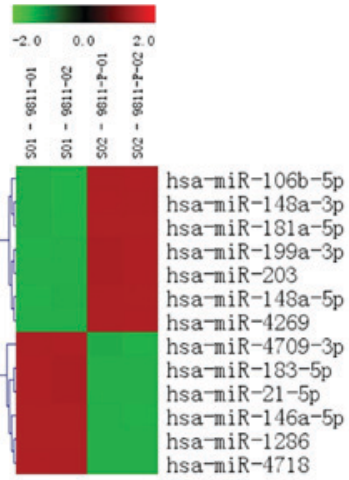

Figure 1. Bioinformatics analysis of miRNAs in gastric cancer cell lines with different peritoneal metastatic potential. (A) A total of 153 miRNAs were identified to be differentially expressed in GC9811-P vs. GC9811 cells, including 74 upregulated and 79 downregulated miRNAs (P<0.05). (B) 13 miRNAs were obtained according to the criteria of signal intensity $>500$ and $\mathrm{P} \leq 0.01$. miRNA/miR, microRNA; hsa, Homo sapiens.
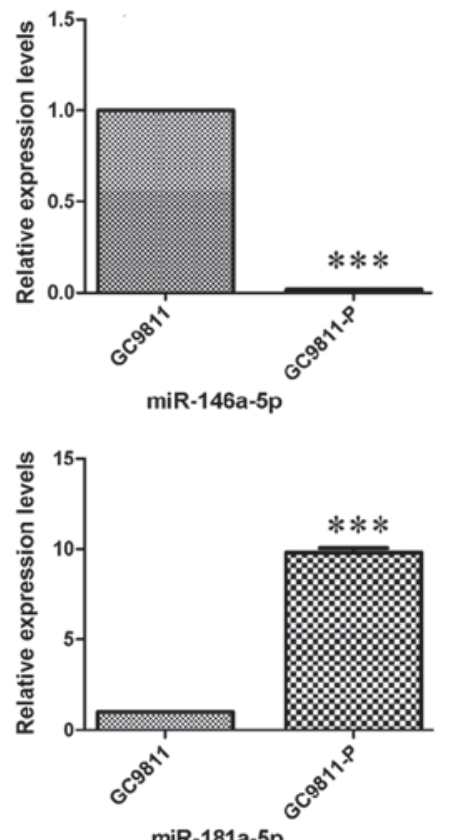

miR-181a-5p

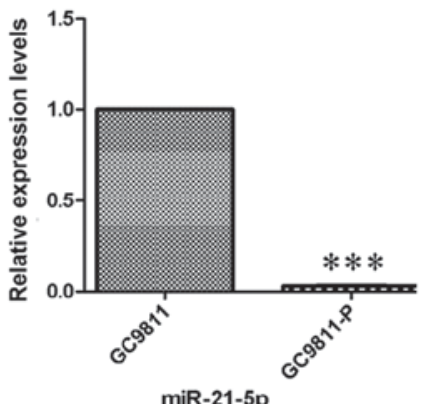

miR-21-5p

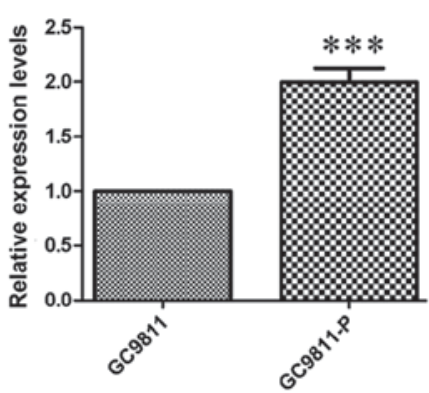

miR-106b-5p

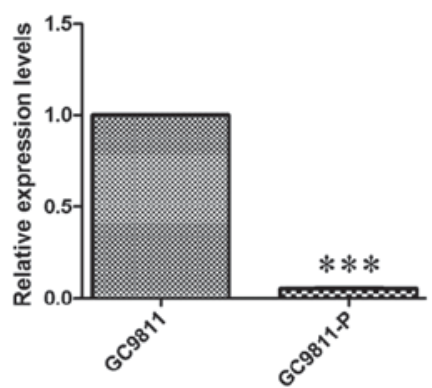

miR-221-3P

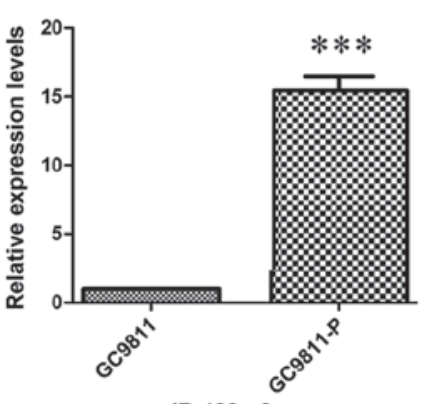

miR-199a-3p
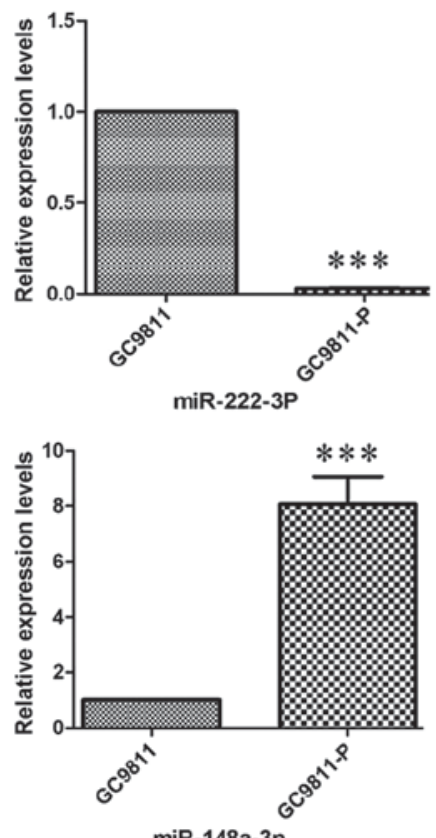

miR-148a-3p

Figure 2. Confirmation of eight deregulated miRNAs in GC9811-P vs. GC9811 cells. Total RNA was extracted from each sample and subjected to reverse transcription-quantitative polymerase chain reaction analysis. All of the experiments were performed in triplicate and the results were normalized to U6 small nuclear RNA 6, pseudogene. ${ }^{* * *} \mathrm{P}<0.001$. miRNA/miR, microRNA. 


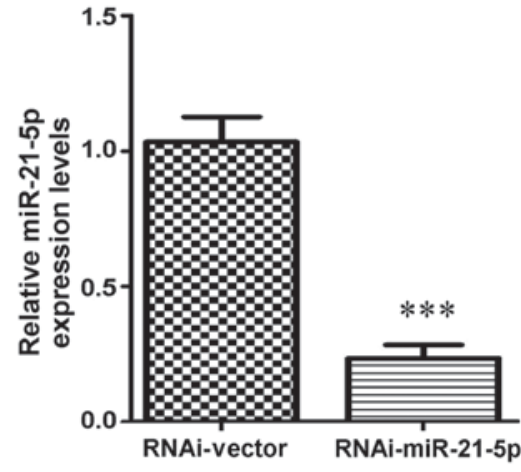

Figure 3. Knockdown of miRNA-21-5P in GC9811 cells. Depletion of endogenous miR-21-5p in GC9811 cells was performed by lentiviral transfection using specific small interfering RNA targeting miR-21-5p or RNAi-Vector. Total RNA was extracted at $48 \mathrm{~h}$ post-transfection and subjected to reverse transcription-quantitative polymerase chain reaction analysis with miR-21-5p-specific primers. Values are expressed as the mean \pm standard deviation Each sample was analyzed in triplicate and normalized to $5 \mathrm{~S}$. ${ }^{* * * *} \mathrm{P}<0.001$ vs. RNAi-Vector group. RNAi, RNA interference; miRNA/miR, microRNA; RNAi-Vector, scrambled control vector.
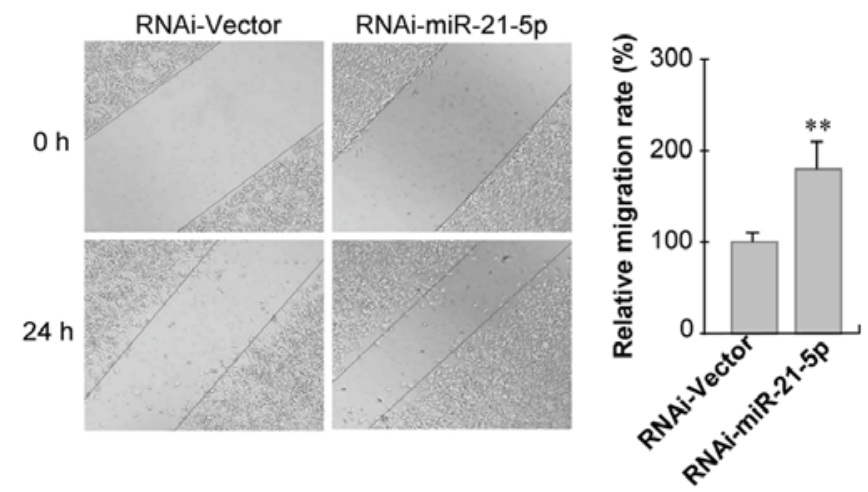

Figure 4. Knockdown of endogenous miR-21-5p increases the migration of GC9811 cells. GC9811 cells with or without knockdown of miR-21-5p were were seeded at a density of $2 \times 10^{5}$ cells per well in 6-well plates. After $12 \mathrm{~h}$, a straight line was scraped. Images were captured under phase-contrast microscopy immediately and $24 \mathrm{~h}$ post-incision (magnification, $\mathrm{x} 100)$. Values are expressed as the mean \pm standard deviation. ${ }^{* *} \mathrm{P}<0.01$ vs. RNAi-Vector group. RNAi, RNA interference; miR, microRNA; RNAi-Vector, scrambled control vector.
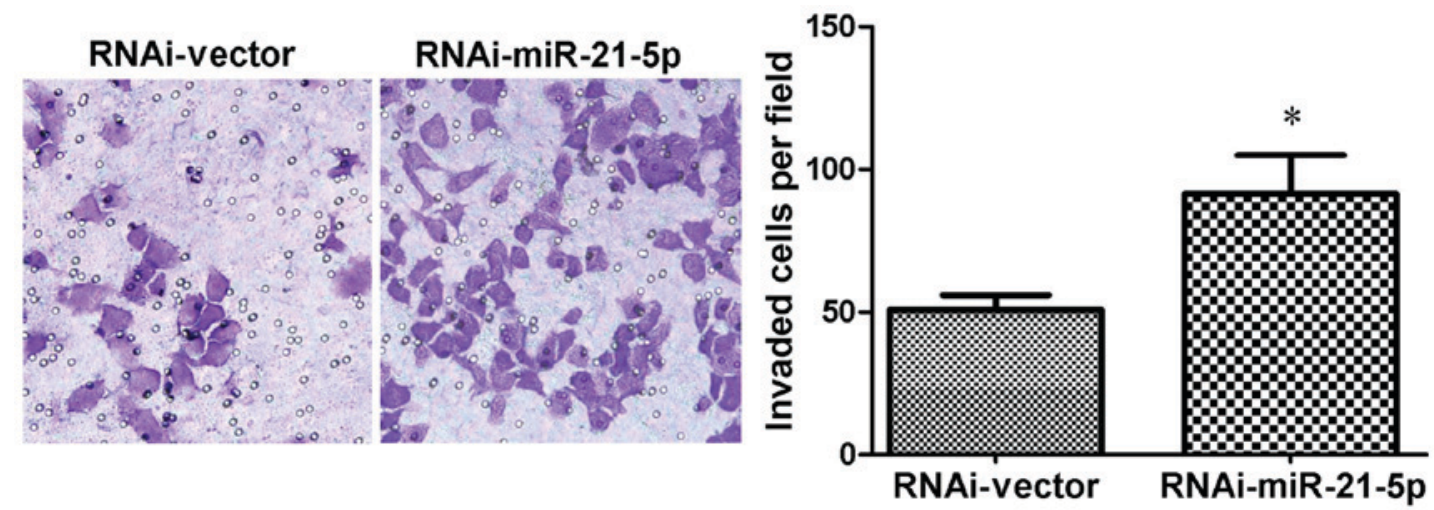

Figure 5. Knockdown of endogenous miR-21-5p promotes the invasiveness of GC9811 cells. GC9811 cells with or without knockdown of miR-21-5p were seeded into the upper chambers of Transwell inserts containing Matrigel-coated micropore membrane filters. Cells that had transgressed the membrane to the lower side were fixed, stained with hematoxylin and counted under the microscope in five fields of view (magnification, x100). A quantitative analysis from three independent experiments is presented in the right panel. Values are expressed as the mean \pm standard deviation. ${ }^{*} \mathrm{P}<0.05$ vs. RNAi-Vector. RNAi, RNA interference; miR, microRNA; RNAi-Vector, scrambled control vector.

metastatic potential using microarray analysis. Among the 13 miRNAs selected according to the criteria of signal intension $>500$ and $\mathrm{P} \leq 0.01,8$ significantly altered miRNAs with fold changes in expression of $>2$ in the GC9811-P cell line as compared with that in the GC9811 cell line were selected. In line with previous studies on other cancer types (14-22), the miRNAs with an altered expression status may be associated with the metastatic potential of gastric cancer.

It is conceivable that aberrant miRNA expression may contribute to the progression of cancer and that these miRNAs may serve as regulators in peritoneal metastasis (23). To this end, the expression level of miR-21-5p, one transcript of miR-21, was further investigated regarding its biological functional relevance to cell invasion and metastasis of gastric cancer. Previous studies have demonstrated that miR-21 may act as an oncogene that is overexpressed and hyperactivated in a variety of cancer types, and that its upregulation promotes the proliferation, apoptosis, invasion and chemoresistance by targeting numerous tumor suppressor genes, including phosphatase and tensin homologue, programmed cell death 4 , tissue inhibitor of metalloproteinases 3 , tropomyosin 1 , ras homolog gene family member B and maspin (24-28). It appears that the majority of studies has considered miR-21-5p a cancer-promoting 'oncomiRs' that drives tumor progression and development $(29,30)$. The present study, however, indicated that downregulated miR-21-5p expression may be associated with the peritoneal metastatic potential of gastric cancer cells. Furthermore, in vitro functional assays demonstrated that silencing of miR-21-3p expression in the native GC9811 cell line markedly enhanced its migratory and invasive potential, suggesting that miR-21-3p may act as a tumor suppressor to prevent gastric cancer peritoneal metastasis. The intrinsic discrepancies between different cancer types and cancer cell lines may be a possible explanation. These results provide novel clues regarding the role of miR-21-5p and indicate its potential clinical value by rendering it a novel target for therapeutic interventions in gastric cancer with peritoneal metastasis.

The target genes of miR-21-5p remain to be completely elucidated. It was speculated that miR-21-5p may inhibit 
gastric cancer metastasis by regulating its specific target genes. A bioinformatics analysis using the MiRanda online prediction tool (http://www.microrna.org/microrna/home.do) identified five genes that are associated with miR-21-5p in the GC9811-P cell line, including stromal antigen 2, nuclear factor $\mathrm{I} / \mathrm{B}$, methylthioadenosine phosphorylase, activity-dependent neuroprotector homeobox and chromodomain helicase DNA binding protein 7 . Based on this, further examination and validation of the precise signaling pathways and molecular mechanisms underlying the involvement of miR-21-5p in the peritoneal metastasis of gastric cancer is warranted.

In conclusion, the present study identified altered miRNA expression patterns associated with peritoneal metastasis in gastric cancer cells. The results also suggested that downregulation of miR-21-5p may be associated with the peritoneal metastatic potential of gastric cancer cells These data further support the notion that altered miRNA expression contributes to the peritoneal metastasis of gastric cancer, and provide molecular biomarkers that may be of therapeutic value for patients with gastric cancer metastasis.

\section{Acknowledgements}

Not applicable.

\section{Funding}

The present study was supported by the National Natural Science Foundation of China (grant nos. 81760440 and 81860426), the Natural Science Foundation of Ningxia, China (grant no. 2018AAC02016), and the Regional Science and Technology Development Program Conducted by the Central Government of China (grant no. YDZX20176400004650).

\section{Availability of data and materials}

The datasets used and/or analyzed during the current study are available from the corresponding author on reasonable request.

\section{Authors' contributions}

YF, FaB, CW, XL and RX performed the experiments, and YF and FaB analyzed and interpreted the data. YY, FeB and $\mathrm{YN}$ conceived the study, designed the experiment and drafted the manuscript. All authors have read and approved the final version of the manuscript.

\section{Ethics approval and informed consent}

Not applicable.

\section{Patient consent for publication}

Not applicable.

\section{Competing interests}

The authors declare that they have no competing interests.

\section{References}

1. Parkin DM, Bray F, Ferlay J and Pisani P: Global cancer statistics, 2002. CA Cancer J Clin 55: 74-108, 2005.

2. Yonemura Y, Endou Y, Sasaki T, Hirano M, Mizumoto A Matsuda T, Takao N, Ichinose M, Miura M and Li Y: Surgical treatment for peritoneal carcinomatosis from gastric cancer. Eur J Surg Oncol 36: 1131-1138, 2010.

3. Bozzetti F, Yu W, Baratti D, Kusamura S and Deraco M: Locoregional treatment of peritoneal carcinomatosis from gastric cancer. J Surg Oncol 98: 273-276, 2008.

4. Wightman B, Ha I and Ruvkun G: Posttranscriptional regulation of the heterochronic gene lin-14 by lin- 4 mediates temporal pattern formation in C. elegans. Cell 75: 855-862, 1993.

5. Lee RC, Feinbaum RL and Ambros V: The C. elegans heterochronic gene lin-4 encodes small RNAs with antisense complementarity to lin-14. Cell 75: 843-854, 1993.

6. Takei Y, Takigahira M, Mihara K, Tarumi Y and Yanagihara K: The metastasis-associated microRNA miR-516a-3p is a novel therapeutic target for inhibiting peritoneal dissemination of human scirrhous gastric cancer. Cancer Res 71: 1442-1453, 2011.

7. Nishida N, Mimori K, Fabbri M, Yokobori T, Sudo T, Tanaka F, Shibata K, Ishii H, Doki Y and Mori M: MicroRNA-125a-5p is an independent prognostic factor in gastric cancer and inhibits the proliferation of human gastric cancer cells in combination with trastuzumab. Clin Cancer Res 17: 2725-2733, 2011.

8. Liang S, He L, Zhao X, Miao Y, Gu Y, Guo C, Xue Z, Dou W, Hu F, Wu K, et al: MicroRNA let-7f inhibits tumor invasion and metastasis by targeting MYH9 in human gastric cancer. PLoS One 6: e18409, 2011.

9. Bai F, Guo X, Yang L, Wang J, Shi Y, Zhang F, Zhai H, Lu Y, Xie H, Wu K and Fan D: Establishment and characterization of a high metastatic potential in the peritoneum for human gastric cancer by orthotopic tumor cell implantation. Dig Dis Sci 52: 1571-1578, 2007.

10. Livak KJ and Schmittgen TD: Analysis of relative gene expression data using real-time quantitative PCR and the 2(-Delta Delta C(T)) method. Methods 25: 402-408, 2001

11. You Y, Liu J, Wang Z, Zhang Y, Ran Y, Guo X, Liu H and Wang H: The enhancement of radiosensitivity in human esophageal squamous cell carcinoma cells by zoledronic acid and its potential mechanism. Cytotechnology 66: 17-25, 2014.

12. You Y, Yang W, Qin X, Wang F, Li H, Lin C, Li W, Gu C, Zhang Y and Ran Y: ECRG4 acts as a tumor suppressor and as a determinant of chemotherapy resistance in human nasopharyngeal carcinoma. Cell Oncol(Dordr) 38: 205-214, 2015.

13. Tseng CW, Lin CC, Chen CN, Huang HC and Juan HF: Integrative network analysis reveals active microRNAs and their functions in gastric cancer. BMC Syst Biol 5: 99, 2011.

14. Liu K, LI G, Fan C, Diao Y, Wu B and LI J: Increased expression of microRNA-221 in gastric cancer and its clinical significance. J Int Med Res 40: 467-474, 2012.

15. Kogo R, Mimori K, Tanaka F, Komune S and Mori M: Clinical significance of miR-146a in gastric cancer cases. Clin Cancer Res 17: 4277-4284, 2011.

16. Li Y, Vandenboom TG II, Wang Z, Kong D, Ali S, Philip PA and Sarkar FH: miR-146a suppresses invasion of pancreatic cancer cells. Cancer Res 70: 1486-1495, 2010.

17. Bhaumik D, Scott GK, Schokrpur S, Patil CK, Campisi J and Benz CC: Expression of microRNA-146 suppresses NF-kappaB activity with reduction of metastatic potential in breast cancer cells. Oncogene 27: 5643-5647, 2008.

18. Hurst DR, Edmonds MD, Scott GK, Benz CC, Vaidya KS and Welch DR: Breast cancer metastasis suppressor 1 up-regulates miR-146, which suppresses breast cancer metastasis. Cancer Res 69: 1279-1283, 2009.

19. Lin SL, Chiang A, Chang D and Ying SY: Loss of mir-146a function in hormone-refractory prostate cancer. RNA 14: 417-424, 2008.

20. Magrelli A, Azzalin G, Salvatore M, Viganotti M, Tosto F, Colombo T, Devito R, Di Masi A, Antoccia A, Lorenzetti S, et al: Altered microRNA expression patterns in hepato-blastoma patients. Transl Oncol 2: 157-163, 2009.

21. Song G, Zeng H, LI J, Xiao L, He Y, Tang Y and Li Y: miR-199a regulates the tumor suppressor mitogen-activated protein kinase 11 in gastric cancer. Biol Pharm Bull 33: 1822-1827, 2010. 
22. Yang H, Kong W, He L, Zhao JJ, O'Donnell JD, Wang J, Wenham RM, Coppola D, Kruk PA, Nicosia SV and Cheng JQ: MicroRNA expression profling in human ovarian cancer: miR-214 induces cell survival and cisplatin resistance by targeting PTEN. Cancer Res 68: 425-433, 2008.

23. Ichimi T, Enokida H, Okuno Y, Kunimoto R, Chiyomaru T, Kawamoto K, Kawahara K, Toki K, Kawakami K, Nishiyama K, et al: Identifcation of novel microRNA targets based on microRNA signatures in bladder cancer. Int J Cancer 125: 345-352, 2009.

24. Feng YH and Tsao CJ: Emerging role of microRNA-21 in cancer. Biomed Rep 5: 395-402, 2016.

25. Motoyama K, Inoue H, Mimori K, Tanaka F, Kojima K, Uetake H, Sugihara K and Mori M: Clinicopathological and prognostic significance of PDCD4 and microRNA-21 in human gastric cancer. Int J Oncol 36: 1089-1095, 2010.

26. Ou H, Li Y and Kang M: Activation of miR-21 by STAT3 induces proliferation and suppresses apoptosis in nasopharyngeal carcinoma by targeting PTEN gene. PLoS One 9: e109929, 2014.

27. Meng F, Henson R, Wehbe-Janek H, Ghoshal K, Jacob ST and Patel T: MicroRNA-21 regulates expression of the PTEN tumor suppressor gene in human hepatocellular cancer. Gastroenterology 133: 647-658, 2007.
28. Wei X, Wang W, Wang L, Zhang Y, Zhang X, Chen M, Wang F, Yu J, Ma Y and Sun G: MicroRNA-21 induces 5-fluorouracil resistance in human pancreatic cancer cells by regulating PTEN and PDCD4. Cancer Med 5: 693-702, 2016.

29. Song Y, Zuo Y, Qian XL, Chen ZP, Wang SK, Song L and Peng LP: Inhibition of microRNA-21-5p promotes the radiation sensitivity of non-small cell lung cancer through HMSH2. Cell Physiol Biochem 43: 1258-1272, 2017

30. Cai L, Wang W, Li X, Dong T, Zhang Q, Zhu B, Zhao H and Wu S: MicroRNA-21-5p induces the metastatic phenotype of human cervical carcinoma cells in vitro by targeting the von Hippel-Lindau tumor suppressor. Oncol Lett 15: 5213-5219, 2018.

This work is licensed under a Creative Commons Attribution-NonCommercial-NoDerivatives 4.0 International (CC BY-NC-ND 4.0) License. 